\title{
Risks Associated With Lentiviral Vector Exposures and Prevention Strategies
}

\section{Citation}

Schlimgen, R., J. Howard, D. Wooley, M. Thompson, L. R. Baden, O. O. Yang, D. C. Christiani, et al. 2016. "Risks Associated With Lentiviral Vector Exposures and Prevention Strategies." Journal of Occupational and Environmental Medicine 58 (12): 1159-1166. doi:10.1097/ JOM.0000000000000879. http://dx.doi.org/10.1097/J0M.0000000000000879.

\section{Published Version}

doi:10.1097/JOM.0000000000000879

\section{Permanent link}

http://nrs.harvard.edu/urn-3:HUL.InstRepos:30371173

\section{Terms of Use}

This article was downloaded from Harvard University's DASH repository, and is made available under the terms and conditions applicable to Other Posted Material, as set forth at http:// nrs.harvard.edu/urn-3:HUL.InstRepos:dash.current.terms-of-use\#LAA

\section{Share Your Story}

The Harvard community has made this article openly available.

Please share how this access benefits you. Submit a story.

\section{Accessibility}




\title{
Risks Associated With Lentiviral Vector Exposures and Prevention Strategies
}

\author{
Ryan Schlimgen, PhD, John Howard, MD, Dawn Wooley, PhD, Maureen Thompson, RN, \\ Lindsey R. Baden, MD, Otto O. Yang, MD, David C. Christiani, MD, MPH, Gustavo Mostoslavsky, MD, PhD, \\ David V. Diamond, MD, Elizabeth Gilman Duane, MS, RBP, CBSP, Karen Byers, MS, RBP, CBSP, \\ Thomas Winters, MD, Jeffrey A. Gelfand, MD, Gary Fujimoto, MD, \\ T. Warner Hudson, MD, and Jatin M. Vyas, MD, PhD
}

\begin{abstract}
Lentiviral vectors (LVVs) are powerful genetic tools that are being used with greater frequency in biomedical laboratories and clinical trials. Adverse events reported from initial clinical studies provide a basis for risk assessment of occupational exposures, yet many questions remain about the potential harm that LVVs may cause. We review those risks and provide a framework for principal investigators, Institutional Biosafety Committees, and occupational health professionals to assess and communicate the risks of exposure to staff. We also provide recommendations to federal research and regulatory agencies for tracking LVV exposures to evaluate long-term outcomes. U.S. Food and Drug Administration approved antiviral drugs for HIV have theoretical benefits in LVV exposures, although evidence to support their use is currently limited. If treatment is appropriate, we recommend a 7-day treatment with an integrase inhibitor with or without a reverse transcriptase inhibitor within 72 hours of exposure.
\end{abstract}

$\mathrm{M}$ ost lentiviral vectors (LVVs) are derived from the human immunodeficiency virus (HIV) type 1 and retain the ability to integrate into the genome of infected cells. ${ }^{1}$ Integrating DNA into the genome of host cells is a powerful technique that allows scientists to manipulate genes and gene expression. ${ }^{2,3}$ This technology is used both at the laboratory bench to answer fundamental questions in biology and in the clinic to provide potential cures to genetic diseases and alter the function of cells that are delivered to patients (eg, chimeric antigen receptor T-cells). ${ }^{4}$ However, this powerful tool also carries the potential to cause oncogenic, infectious, and other transformative changes to infected cells.

From the Partners HealthCare, Boston, Massachusetts (Dr Schlimgen); National Institute for Occupational Safety and Health, Washington, District of Columbia (Dr Howard); Wright State University, Detroit, Michigan (Dr Wooley) Emory University, Atlanta, Georgia (Ms Thompson); Dana Farber Cance Institute (Dr Baden, Ms Byers); Brigham and Women's Hospital (Dr Baden); Harvard Medical School, Boston, Massachusetts (Drs Baden, Christiani, Gelfand, Vyas); University of California Los Angeles, Los Angeles, California (Drs Yang, Hudson); Massachusetts General Hospital (Drs Christiani, Gelfand, Vyas); Harvard TH Chan School of Public Health (Dr Christiani); Boston University School of Medicine, Boston (Dr Mostoslavsky); Massachusetts Institute of Technology, Cambridge (Dr Diamond); Environmenta Health and Engineering (Ms Duane); Occupational and Environmental Health Network, Boston, Massachusetts (Dr Winters); Occupational Medicine consultant, Los Altos, California (Dr Fujimoto).

The conference and preparation of this manuscript was supported in part by an unrestricted grant from the Eagleson Institute.

Authors Vyas, Schlimgen, Howard, Wooley, Thompson, Baden, Yang, Christiani, Mostoslavsky, Diamond, Duane, Byers, Winters, Gelfand, Fujimoto, and Hudson have no relationships/conditions/circumstances that present potentia conflict of interest.

The JOEM editorial board and planners have no financial interest related to this research.

Address correspondence to: Jatin M. Vyas, MD, PhD, Massachusetts General Hospital, 55 Fruit St, GRJ-5-504, Boston, MA 02114 (jvyas@ partners.org).

Copyright (C) 2016 American College of Occupational and Environmental Medicine

DOI: 10.1097/JOM.0000000000000879

\section{Learning Objectives}

- Become familiar with the potential harms associated with exposure to lentiviral vectors (LVVs)

- Summarize the authors' proposed framework for researchers, institutional safety committees, and occupational health professionals relevant to LVVs.

- Discuss the authors' recommendations for dealing with LVV exposures, including the supporting evidence.

The oncogenic potential of LVVs can manifest through multiple mechanisms. LVV-infected cells can become cancerous through activation of oncogenes or inactivation of tumor suppressor genes. Insertional mutagenesis results from gene dysregulation at the site of LVV integration within or near a coding region of the host genome. Such integration can cause outright gene disruption or overexpression of normal cellular genes due to insertion of a nonphysiologic promoter. Another pro-oncogenic mechanism is gene transduction of an oncogenic transgene. In addition, designed LVVs can target multiple genes and regulatory pathways simultaneously (eg, libraries of shRNA or CRISPR sgRNA), which could effectively silence or inactivate tumor suppressor activity. Finally, the unknown functions of some transgenes may promote oncogenesis or other toxic effects. Oncogenic changes created by these mechanisms will be carried by the infected cell and its progeny.

Another concern of LVVs derived from HIV is the possibility that recombination unintentionally reconstitutes a replication-competent and pathogenic virus. The design of LVV constructs has evolved to increase the carried transgene size and reduce the risk of developing a replication-competent virus (Fig. 1). ${ }^{5}$ The identification of HIV genes not required for integrating the transgene has permitted the engineering of the multiple-attenuated packaging systems. ${ }^{6,7}$ In second-generation LVVs, four accessory genes, vif, $v p r, v p u$, and nef, are removed without major negative effects on vector yield or infection efficiency (Fig. 1C). ${ }^{6,8,9}$ These modifications also improve LVV safety, as any lentivirus that becomes replication-competent would be devoid of these virulence factors. Therefore, first and second-generation LVVs reduce, but do not eliminate the probability of creating pathogenic replication-competent viruses. The design of LVVs is further refined in the third and fourth generations by separating the genes necessary for creating the LVV onto additional plasmids (Fig. 1D and E).$^{10,11}$ Moreover, some vectors include self-inactivating sequences that significantly reduce expression from the viral promoter after integration. ${ }^{5,12}$ This design has substantially reduced, but not eliminated the risk of unintentionally generating replication-competent viruses. ${ }^{13}$

Still, this design does not eliminate the risks of insertional mutagenesis and the intrinsic risks associated with the transgenes inserted by the viral vector. Risk of exposure that promotes infection in a research worker is mitigated in the laboratory by eliminating the 

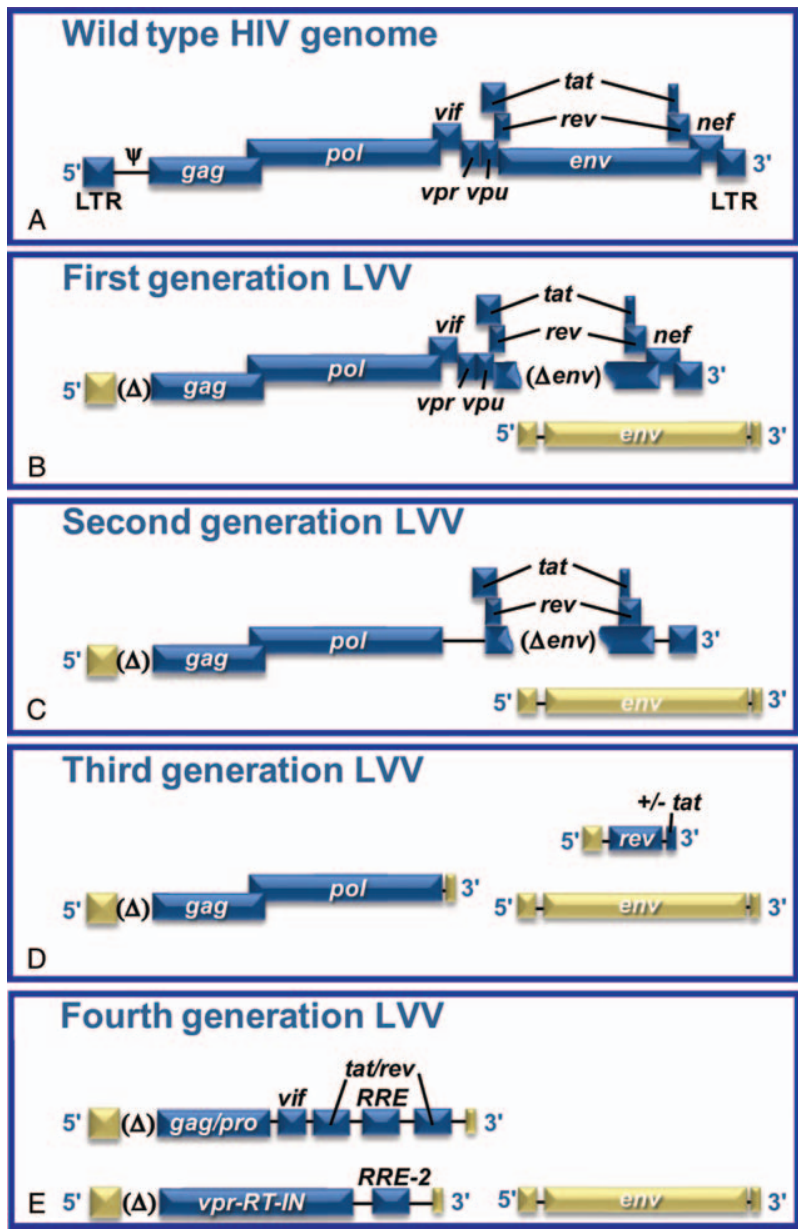

FIGURE 1. The development of LV packaging systems from HIV. A, Wild-type HIV genome with all of its genes and regulatory elements provides the backbone for LVVs. B, First-generation LVVs removed the envelope protein and the psi packaging signal and incorporated a heterologous promoter to reduce recombination potential. C, Second generation of LVV removed accessory genes (vif, vpr, vpu, and nef) to reduce the virulence of any potential replication-competent retrovirus. D, Third-generation LVV eliminated the transactivator gene, tat, and split the vector into three plasmids to reduce further recombination potential, retaining only the three genes necessary for transgene expression (gag, pol, rev). E, Fourth-generation LVV split the gag and pol onto separate plasmids to reduce even further recombination potential. This generation added back some HIV genes to enhance transduction efficiency and transgene expression.

use of sharps wherever feasible, and implementing extrinsic biocontainment strategies and personal protective equipment (PPE). Moreover, experimental procedures can be modified to reduce the concentration and volume of LVV to minimize the impact of a potential exposure. In this paper, we review the potential risks associated with LVV exposure and provide recommendations for principal investigators (PIs), Institutional Biosafety Committees (IBCs), and occupational health professionals to prevent and prepare for potential exposures, including guidelines for LVV exposure response plans. We also provide recommendations to federal research and regulatory agencies for tracking LVV exposures in order to evaluate long-term outcomes.

\section{RISKS OF LENTIVIRUS AND TRANSGENE EXPOSURES}

LVVs are designed to deliver and integrate genes into the genome of a cell and are being tested clinically to treat genetic disorders and endow cells with specific functions. ${ }^{14}$ These trials have provided some evidence of the risks associated with viral vectors. In two early gene therapy trials, 20 children with X-linked severe combined immunodeficiency (SCID) were treated using Moloney murine retrovirus-derived vectors, a viral vector that integrates into host DNA, similar to LVVs. Of those 20 children, five individuals developed leukemia due to insertional mutagenesis. ${ }^{15}$ Indeed, Moloney murine retrovirus-derived viral vectors have different integration profiles than HIV, the backbone for LVVs. Additional studies support a link between viral vector systems and oncogenesis. In the first study, two of 10 patients with X-linked SCID developed uncontrolled exponential clonal proliferation of mature $\mathrm{T}$ cells, presumably due to retrovirus vector integration in proximity to the LMO2 proto-oncogene promoter. ${ }^{16}$ In the second study, one of 10 patients treated with a murine gamma-retroviral vector to correct X-linked SCID developed clonal T cell acute lymphoblastic leukemia (T-ALL) as a result of insertional mutagenesis. ${ }^{17}$ Together, these studies show that modified murine leukemia viruses present a high risk of leukemogenesis as a result of their powerful enhancer sequences located in the intact LTR regions. In another study, two patients who underwent lentiviral treatment for X-linked chronic granulomatous disease progressed to myelodysplasia (15 months postinfusion) or acute myeloid leukemia (28 months postinfusion), due to insertional activation of oncogenes and silencing of tumor suppressor genes. ${ }^{18}$ Finally, a patient treated with LVV for beta-thalassaemia developed a nonmalignant, clonal expansion of erythroblasts due to integration of a LVV transgene into the HMGA2 locus. ${ }^{19}$ This expansion was limited to erythroblasts presumably because of the tissue specificity of the $\beta$-locus control region used to drive expression of the $\beta$-globin gene. It is unknown at this time if the clone will remain homeostatic or serves as a prelude to oncogenic transformation. ${ }^{19}$ Thus, LVVs can cause clonal expansion and may cause oncogenesis through insertional mutagenesis when given purposely in high doses with the intention to modify DNA of human subjects. And, there is a potential risk of accidental insertional mutagenesis for an individual inadvertently exposed to LVV engineered for research purposes.

The potential risks from LVVs are dependent upon the nature of the exposure. LVVs are derived from blood-borne pathogens that generally require direct contact with either blood or mucous membranes to initiate infection. In the laboratory, the majority of exposures to LVVs occur by direct contact with intact skin. As with HIV, exposure to intact skin is not clinically relevant and does not pose a significant risk. The preferred method to respond to this exposure is copious washing of the affected area with running water to dilute, cleanse, and flush the LVVs from intact skin. There is no indication for postexposure chemoprophylaxis for an intact skin exposure. Clinically relevant exposures result through parenteral inoculations, contact with the mucous membranes of the eyes, nose, or mouth, or through direct contact with nonintact skin. The best methods to prevent exposure are proper technique, training, and personal protection equipment. This will include carefully reviewing the use of sharps in laboratory procedures and eliminating them when possible: for example, plastic substitutes for glass Pasteur pipettes are commercially available. Needle/syringes should not be used. $^{20}$

Indirect contact with droplets or smaller airborne particles may occur in the laboratory setting. Aerosol exposures through droplet transmission are another potential route of LVV exposure. The NIH Healthcare Infection Control Practices Advisory Committee (HICPAC) defines droplet transmission as an indirect contact 
route that results from infectious agents depositing on susceptible mucosal surfaces of the recipient, generally over short distances. In contrast, HICPAC defines airborne transmission as the dissemination of infectious agents in the respirable size range that remain infective over time and distance. ${ }^{21}$ Aerosol-generating procedures (such as centrifuging or pipetting) with LVVs can generate droplets that are generally 5 to $10 \mu \mathrm{m}$ in diameter. ${ }^{22}$ These droplets will fall out of the air in $\sim 15$ to 67 minutes. Pedrosa and Cardoso ${ }^{23}$ have shown a $30 \%$ penetration of $5 \mu \mathrm{m}$ particles to the alveoli and $50 \%$ penetration of $10 \mu \mathrm{m}$ particles to the tracheobronchial region. There is no significant penetration of particles $20 \mu \mathrm{m}$ or greater beyond the trachea. There have been no documented cases of airborne transmission of HIV in clinical laboratories handling patient specimens; however, droplet transmissions have been reported. ${ }^{24}$

These data support treating droplet contact with mucous membranes (droplet transmission), but not airborne transmission, as a clinically relevant exposure. In addition, as LVVs are often designed to infect a broader range of human cells than HIV [eg, replacing the HIV envelope with vesicular stomatitis virus envelope glycoprotein (VSV-G)], the entire mucosal membrane of the tracheobronchial region can potentially be infected. Thus, droplet transmission poses a risk hazard and aerosol-generating procedures with LVVs should be performed in primary containment (ie, biosafety cabinet or sealed centrifuge rotors). In summary, clinically relevant LVV exposure is limited to individuals who have experienced (1) direct parenteral inoculation, (2) contact with mucous membranes or nonintact skin, or (3) direct contact at a close range to droplets from an aerosol-generating procedure outside of primary containment.

\section{RECOMMENDATIONS FOR OCCUPATIONAL HEALTH PROFESSIONALS}

Use of antiretroviral drugs has extended from primary treatment of HIV infection to postexposure prophylaxis (PEP) for highrisk exposure. Considering the potential risks of LVVs versus the generally favorable safety profiles of newer anti-HIV drugs, we recommend that occupational health professionals consider offering PEP after a clinically relevant LVV exposure following a discussion with the exposed individual of the risks and benefits of antiretroviral therapy. Although there are no direct data to support this recommendation, we believe that experience with HIV PEP provides some guidance. Ideally, the laboratory staff, the PI, and the occupational health professionals should review the LVVs being used and develop a laboratory-specific exposure response plan before an exposure incident (see Appendix 1 for a sample exposure response plan.) This exposure plan should be available to the responding clinician when a clinically relevant exposure occurs. Review and approval of such research is often a function of the IBC. Knowledge of the LVVs being used and an established exposure response plan allow involved staff to consider carefully the risks and benefits of PEP. If the decision is made to treat an exposed individual, there are no data to support a specific regime or duration of prophylaxis.

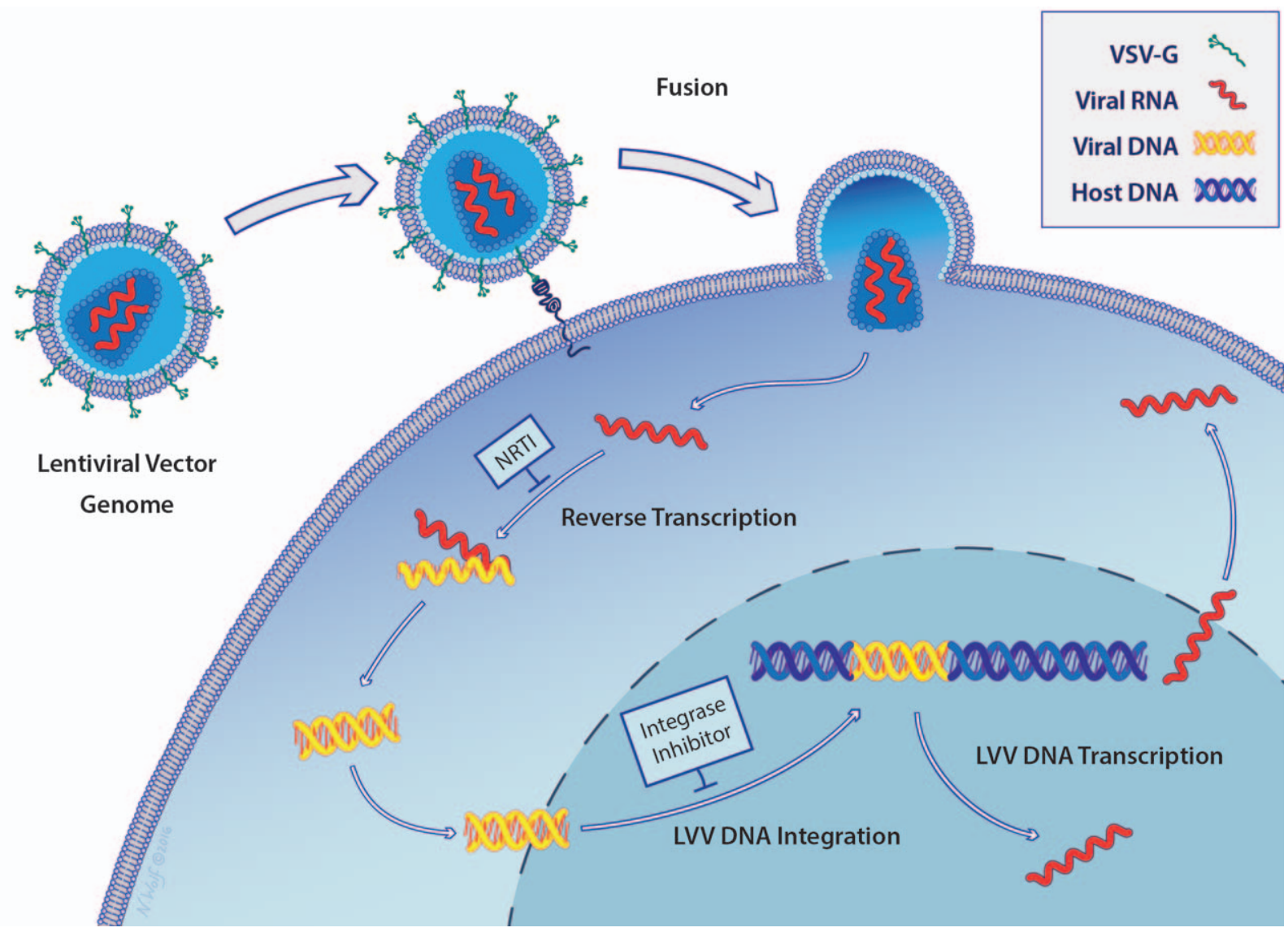

FIGURE 2. Mechanism of nucleic acid delivery by LVVs. Viral particles containing lentiviral RNA fuse to the target cell, releasing the LVV RNA into the cell. Reverse transcription converts the LVV RNA to DNA, which integrates into the host genome and is expressed by the infected cell. After integration, the cell can become cancerous through LVV transgene expression or insertional mutagenesis. Reverse transcription can be blocked by NRTIs [nucleos(t)ide reverse transcriptase inhibitors] and LVV DNA integration can be blocked with HIV integrase inhibitors. Illustration by Nicole Wolf, MS, ®2016. Printed with permission. 
However, on the basis of the basic biology of LVVs, we recommend the use of an integrase inhibitor with or without a reverse transcriptase inhibitor. There are no data regarding the optimal number of drugs for PEP, but given the theoretical risks of LVV exposure versus the small but concrete risks of antiretroviral drugs, some favor single-drug PEP with the integrase inhibitor (which has the best safety profile).

An examination of the HIV infection cycle reveals why some current antiretroviral treatments would potentially treat LVV exposures (Fig. 2). Current antiretroviral drugs for HIV infection include nucleos(t)ide reverse transcriptase inhibitors (NRTIs), nonnucleoside reverse transcriptase inhibitors (NNRTIs), protease inhibitors, fusion inhibitors, entry inhibitors (CCR5 coreceptor antagonist), and HIV integrase strand transfer inhibitors. Of these treatments, only NRTIs and HIV integrase strand transfer inhibitors act upon pathways retained in LVVs, and other drug classes would provide no benefit for PEP. LVVs are usually pseudotyped with a heterologous envelope protein, thus eliminating HIV-1 specific entry and fusion inhibitors as effective therapies. LVVs do not contain genes for replication and expression of the Gag pre-protein, thus viral protease inhibitors would not be effective.

The discussion between the occupational health professional and the exposed individual should include the potential risks of insertional mutagenesis and oncogenesis, emphasizing that there have been no definitive case reports of such harm from occupational exposures. However, these adverse effects may be difficult to detect because they may appear years after the exposure and be masked as a naturally occurring cancer. The conversation should also note that the proposed treatment is based on accepted HIV PEP guidelines from the Centers for Disease Control and Prevention, ${ }^{25}$ and demonstrated to reduce the risk of HIV infection (the parent virus) after exposure, but has not been clinically proven to prevent insertional mutagenesis from an integrated LVV. This discussion should be balanced with the inherent risks of antiretroviral medications. Current therapies have a tolerable side effect profile and are currently prescribed prophylactically to healthy individuals at a high risk for HIV exposure [pre-exposure exposure prophylaxis $(\mathrm{PrEP})]{ }^{26}$ The risk tolerance of the exposed individual is a critical factor in the decision to treat, as the benefits of PEP are unknown at this time. If a decision is made to treat the individual with antiretroviral PEP, we recommend a 7-day treatment with an integrase inhibitor with or without a NRTI to commence as soon as possible postexposure but within 72 hours of exposure (Table 1). Current PEP for occupational HIV exposure recommends 28 days of treatment, ${ }^{25}$ but there are important differences between LVVs and HIV. Both third and fourth-generation LVVs have a low risk of creating a replication-competent virus in contrast to HIV, which is intrinsically replication-competent. Second, only the first round of integration for LVVs poses risk. However, as LVVs cannot replicate, the risk beyond integration is thought to be very low. Integration should take place within hours of exposure, so longer treatments beyond 7 days may not provide additional benefits. Finally, more drugs are generally chosen for HIV PEP because the drug resistance profile is generally unknown, and there are significant rates of single and even double drug class resistant viruses in circulation. ${ }^{25}$

TABLE 1. Proposed Lentiviral Vector Postexposure Prophylaxis

Initiation of treatment Duration of treatment

Types of treatment

\author{
0-72 hours after exposure \\ 7 days \\ Integrase inhibitor-eg, raltegravir \\ With or without \\ Nucleoside reverse transcriptase \\ inhibitor (NRTI)—eg, tenofovir
}

Treatment should be started as soon as possible, as the primary goal of therapy is to prevent the LVV integration event. Maximum benefit would be theoretically achieved if treatment is started immediately or within 2 hours. After 72 hours, there is likely no benefit to treatment, as any LVVs present in the individual will have either entered the cell or have been cleared. As a quick response is paramount to treatment, it is advisable to start treatment and then discontinue rather than delay initiation of treatment. Because it is foreseeable that the risk-benefit discussion will often be protracted, we strongly recommend developing an exposure response plan during the IBC review and experimental design discussion.

For most LVV exposures, an integrase inhibitor (eg, ralegravir or dolutegravir) with or without a NRTI (eg, tenofovir) could be considered. The integrase inhibitors have an increasingly acceptable safety and tolerability profile in the treatment of HIV infection. Tenofovir is also considered generally safe and well-tolerated (used extensively for HIV and HBV treatment) but can result in nephrotoxicity and an extremely rare risk of fatal lactic acidosis. Before using these medications, an assessment for pre-existing HIV or HBV infection should be made to minimize the chances of inducing antiviral resistance. Both an integrase inhibitor and a NRTI are components of recommended first-line therapies for HIV and HIV $\mathrm{PEP}^{25,26}$ In appropriate cases, we recommend the use of these drugs with the understanding that they have not been approved for these indications by the FDA. The short duration of treatment reduces the risk for side effects. However, when determining the risk tolerance of the exposed individual, single drug therapy should be considered if there is a contraindication to one of the drug classes. Drug combinations are used for HIV PEP to prevent and circumvent possible pre-existing viral drug resistance ${ }^{25}$; however, resistance is not a significant concern for LVV exposure because nearly all LVVs use a native HIV-1 backbone, which should be fully drug susceptible. Thus, the benefit of using multiple drugs to treat LVV clinically relevant exposure is unclear, but would theoretically derive from the additive benefit of each drug alone.

Occupational health programs should develop a baseline LVV exposure response plan that could be initiated quickly when an exposure incident is reported. The baseline LVV exposure control plan should be maintained at the institution. In addition to a baseline exposure response plan, a laboratory-specific protocol should be developed with the help of the IBC, the institutional Biosafety Officer (BSO), and the PI that considers details of LVVs used in the laboratory. This effort will require the IBC to identify all registrations and laboratories that use LVVs and provide the relevant information to occupational health programs. This information and decision criteria for modifying an exposure response plan will be discussed in more detail in the next section. The laboratory-specific exposure response plan, depending upon the organizational structure of the institution, may be best kept by the PI in the laboratory, the BSO, the IBC, and/or in the occupational health program. Thus, when an individual arrives at occupational or employee health clinic reporting an LVV exposure incident, the first step would be to identify the laboratory-specific response plan. If this is not readily accessible, the default institutional response protocol could be initiated in the first 2 hours, while the laboratory-specific plan is located (or developed) and the ongoing PEP modified accordingly. In situations wherein an institution does not have an occupational health clinic/provider, guidance on management of these exposures should be generated by the institution and given to the affected laboratory worker to present to the Primary Care Physician or Emergency Department personnel.

Finally, there is no "right" approach for treating LVV exposure as treatment currently employed for laboratory exposures range from no treatment to administration of antiretrovirals. The uncertainty stems from being unable to quantify the potential risk of insertional mutagenesis from LVV exposure. It balances the LVV 
risks against the risks and theoretical benefits of treatment. Occupational health professionals should use caution when presenting the potential benefits of PEP treatment, but not dismiss the potential risks associated with LVV.

\section{RECOMMENDATIONS FOR INSTITUTIONAL BIOSAFETY COMMITTEES}

The IBC has two important roles to ensure the safety of the laboratory staff and clinicians working with LVVs. First, the IBC is responsible for assuring that the PI understands and communicates the risks to laboratory personnel who work directly with the biological materials. Second, the IBC acts a source of information for occupational health professionals during an exposure incident. This information can be formalized in an exposure response plan and should be included in a biological safety/IBC registration. Developing an exposure response plan should be a joint effort between the PI, BSO, occupational health, and the IBC. Moreover, the inclusion of occupational health professionals on the IBC (either as voting members or as ex-officio members) can strengthen this relationship and ensure that the IBC provides the necessary information to the health care provider evaluating the exposed individual. The modern biological laboratory comprises many individuals who work outside traditional hours (ie, 9:00 AM to 5:00 PM). Exposed laboratory workers often have incomplete information about the critical details of the LVV to make timely clinical decisions. Following an exposure incident, the PI should be able to provide critical information regarding the nature of the LVV. When the PI is unavailable, the BSO or IBC contact can relay this critical information to the occupational health clinic. Therefore, the IBC needs to collect critical information about the use of LVVs from the PI before an incident occurs. Further, the IBC must ensure that the PI is aware of the risks associated with LVVs and has developed an LVV exposure response plan that is readily available in the event of an exposure. Ideally, the exposure response plan should be readily available in the laboratory for any exposed researcher to access and present to a clinical facility for postexposure evaluation and possible treatment.

IBCs should also require PIs, as part of the review and approval process of LVV registrations, to develop laboratoryspecific exposure response plans. Investigators should reach out to their institutional BSO and occupational health professionals to assist with developing the laboratory specific LVV exposure response plan. These plans should be provided in a succinct document that includes at least the following information. First, it should include the appropriate work practice controls and PPE for procedures involving LVVs. Second, the plan should outline the actions an exposed individual and their PI should take in immediate response to the different types of LVV exposure. For example, in the event of an ocular exposure, the eye (s) should be flushed at an eyewash station for 5 to 15 minutes. Third, the plan should include contact information for the institution's occupational or employee health clinic and instructions to report within 2 hours of an exposure. In addition, it should be emphasized that antiretroviral drugs may be offered to individuals exposed to LVVs who meet the definition of a clinically relevant exposure. However, the final decision to offer antiretrovirals will be determined by potential risk of the exposure, a clinical determination made by the occupational health professional. Finally, in consultation with occupational health professionals, the IBC, and the PI, drug treatments may be listed in the exposure response plan that are specific to the LVV being used in the laboratory that considers the origin of the vector and transgene (s) being carried by the vector.

Once the information is collected and laboratory-specific exposure response plan developed, the PI or supervisor should discuss potential risks with every individual in the laboratory working with LVVs. The discussion should include each element of the laboratoryspecific exposure response plan. The PI should emphasize that PEP may be offered to the individual by occupational health personnel in the event of a clinically relevant exposure. The IBC should obtain written verification from the PI that they have adequately described the exposure response plan to each individual in their laboratory and that individuals working with LVVs understand that PEP may be offered after a clinically relevant exposure. Similar protocols should be followed in animal facilities that use LVVs.

The NIH Guidelines for Research Involving Recombinant or Synthetic Nucleic Acid Molecules (NIH Guidelines) require that PIs receive IBC approval of all LVV work before their use in animal models, and at a minimum, PIs notify the IBC of all LVV work before their production or use in immortalized cell lines. ${ }^{27}$ In institutions that do not receive federal funding, it is best to follow these federal guidelines. There is a range of information that institutions currently require PIs to provide before IBC review and approval. To identify and categorize the risk of the LVVs, we recommend that IBCs require investigators to submit detailed information concerning the viral vector being used in the laboratory as well as the nature of the transgene inserted into the LVV, as denoted in Table 2.

TABLE 2. Information to Help the Risk Assessments of Viral Vectors

\begin{tabular}{|c|c|c|}
\hline Information Provided to IBC & Low-risk Examples & Clinically Relevant Examples \\
\hline Transgene function & Protein-based fluorescence (eg, GFP) & $\begin{array}{l}\text { Silence a tumor-suppressor or express an } \\
\text { oncogene (ie, Ras, } M y c \text {, etc.) }\end{array}$ \\
\hline Number of plasmids used to generate virions & 3-4 plasmids & 2 or less plasmids \\
\hline Mutations within LVVs & $\begin{array}{l}\text { LVVs that use self-inactivating long } \\
\text { terminal repeats (LTRs) and other } \\
\text { deleterious mutations }\end{array}$ & Wild-type LTRs \\
\hline Expression control elements & Weak promoters & $\begin{array}{l}\text { Strong promoters present (CMV, SV } 40 \text {, } \\
\text { etc.) }\end{array}$ \\
\hline Host range & Nonhuman tropism & Expanded host range (ie, VSV-g) \\
\hline Concentration & $<1 \times 10^{9}$ infectious units $/ \mathrm{ml}$ & $>1 \times 10^{9}$ infectious units $/ \mathrm{ml}$ \\
\hline Production volume & $<100 \mathrm{~mL}$ & $>100 \mathrm{~mL}$ \\
\hline Percentage of genome deleted or substituted & $>2 / 3$ & $<2 / 3$ \\
\hline Vector name & \multicolumn{2}{|c|}{ pCI-VSVg, pRSV-Rev, pMD2.G, etc. } \\
\hline $\begin{array}{l}\text { Name and provider of the transgene or target } \\
\text { sequence }\end{array}$ & \multicolumn{2}{|c|}{ Commercial (Addgene, etc.) or Academic (Scientific Collaborator or Core Facility) } \\
\hline $\begin{array}{l}\text { Site of generation (eg, laboratory, core facility, } \\
\text { or commercial provider) }\end{array}$ & \multicolumn{2}{|c|}{ PI's laboratory, institutional core facility, or commercial provider } \\
\hline
\end{tabular}


This information is critical for the IBC and for occupational health professionals in order to counsel and treat an exposed individual. The name and provider of the target sequence identifies the transgene and allows the risks associated with the transgene to be reviewed by the IBC. The function of the transgene, if known, is important for determining whether the transgene has inherent oncogenic potential. The vector name and type is important, especially identifying the generation of LVV, as some vector backbones are by design resistant to therapeutic drugs and may modify the PEP offered to exposed individuals deemed eligible for antiretroviral therapy. Identification of expression control elements will highlight strong and weak promoters and enhancers that may indicate higher risk. Host range is also important, as many LVVs are pseudotyped with VSV-g to allow for transduction of a broad range of mammalian cells. ${ }^{28}$ Using an envelope protein with a narrow host range and tissue specificity will reduce the risk associated with LVV exposure. High concentration (greater than $1 \times 10^{9}$ infectious units/ $\mathrm{mL}$ ) increases the risks of exposure when compared with lower concentrations. $^{2}$ In addition, working with large volumes of LVVs (greater than $100 \mathrm{~mL}$ ) produces a higher risk. There are higher risks associated with the process of generating LVVs than using them in a transduction assay, so knowledge of the site of generation will identify those individuals at a higher risk. Finally, not only stating the percentage of viral genome present in an LVV is required by the NIH Guidelines ${ }^{27}$ but also serves as a check for determining the LVV generation: third and fourth-generation LVVs should have less than two-thirds of the HIV viral genome.

\section{RECOMMENDATIONS FOR FEDERAL RESEARCH AND REGULATORY AGENCIES}

If an individual is exposed to a LVV, state and local governments may require notification. At the federal level, the NIH Office of Biotechnology Activities (OBA) must be notified of all overt exposures to recombinant and synthetic nucleic acid molecules from all institutions that receive federal funding. ${ }^{27}$ The federal government records the incident only and specifically avoids recording the name of the exposed individual. The Occupational Safety and Health Administration (OSHA) require all blood-borne pathogen exposures from needle sticks and cuts from sharps be recorded by the employer on the OSHA Log of Work-Related Injuries and Illnesses (OSHA Form 300). Thus, the only entity tracking and following up with exposed individuals is the institution itself.

NIH OBA incident reports are required to include a description of the nature, cause, and consequences of the exposure incident. The institution completes a report that includes the measures that it took in response to mitigate the root cause of exposure and prevent reoccurrence. The institution needs to identify the PI as well as any medical surveillance provided or recommended after the incident and if there was an injury or illness associated with the incident. OSHA regulations do not explicitly mention lentiviruses, but could potentially cover exposure incidents through the Bloodborne Pathogens (BBP) Standard, the recording criteria for needle stick and sharps injuries (29 CFR Section 1904.8), and the general duty clause (29 USC Section 654). ${ }^{20}$ The general duty clause of the Occupational Safety and Health Act requires each employer to "furnish to each of his employees employment and a place of employment which are free from recognized hazards that are causing or are likely to cause death or serious physical harm to his employees." ${ }^{29}$ Only the BBP Standard offers any specific requirements for handling human source materials, while the other standards and regulations provide justifications for investigations, fines, and corrective actions after an incident has been reported and investigated by OSHA.

Collecting and tracking information from individuals to evaluate the long-term risks associated with lentiviruses is currently managed by the institution. This is a difficult task for most institutions. First, there may not be an immediate host response to an exposure. Long latency health effects are more difficult to trace back to an exposure occurring years before and may create a false sense of risk avoidance. Second, the individuals most likely to be exposed to LVVs are a highly mobile population of undergraduate students, graduate students, postdoctoral scientists, technicians, nurses, and research physicians who are not likely to stay with the laboratory or the institution for the 10 to 20 years necessary to observe possible signs and symptoms of the exposure. Third, there is no standard method or guidance for institutions to collect and track information about exposure incidents, so the information is not uniformly collected, stored, or analyzed.

Occupational exposure registries are one method to track potential long-term effects of exposure to LVVs. Surveillance registries of workers who are occupationally exposed to LVVs may be helpful in determining the incidence and prevalence of any potential adverse effects from LVV exposure incidents. Occupational exposure registries have been used to track workers exposed to ionizing radiation, asbestos, beryllium, and toxins from the World Trade Center disaster. ${ }^{30}$ In addition, the National Institute for Occupational Safety and Health (NIOSH) Nanotechnology Research Center (NTRC) supports epidemiologic studies for nanomaterial workers, including medical, cross-sectional, prospective cohort, exposure studies, and exposure registries. ${ }^{31,32}$

We recommend that the federal government through either the CDC or OBA establish a registry to follow LVV-exposed individuals and support epidemiologic studies for scientific researchers working with lentiviruses, including medical, crosssectional, prospective cohort, and exposure studies to assess any long-term effects of lentivirus exposures.

\section{CONCLUSION}

Preventing exposures to LVVs is an important element in occupational health and biosafety programs. Incident reports should capture details on the procedure being conducted; this allows review of the prevention efforts. Most exposures involve intact skin and can be handled with flushing of affected area with copious amounts of water. Other clinically relevant exposures that merit consideration for antiretroviral prophylaxis include (1) direct parenteral inoculation; (2) contact with mucous membranes or non-intact skin; and (3) direct contact at a close range to droplets from an aerosolgenerating procedure outside of primary containment. Treatment of LVVs exposure poses difficult questions because of the need to balance potential risks with these medications that carry inherent risks and potential benefit to prevent infection with LVVs. To address these difficult questions, we propose a three-pronged approach to treatment of LVVs.

The IBC, PIs, and personnel working with LVVs should weigh the risks and potential benefits of treating clinically relevant exposure incidents before the event. IBCs should provide the support, guidance, and training to the PI so that they can have discussions with their staff at the time of experimental design when the decision is made to use these powerful genetic tools. Upon approval, the IBC should include recommendations for offering or deferring PEP, with rationale based on the risk of material. This information should be recorded in the registration under the occupational medicine section. Occupational health providers and laboratory personnel should then weigh these risks to determine an individual's preference for treatment if an exposure was to occur.

If the decision is made to treat an exposure, an immediate short course of an integrase inhibitor and NRTI can be used as PEP.

The federal government should track and collect information from LVV-exposed individuals to evaluate the long-term risks associated with lentiviruses, as they are uniquely suited to collect this information.

As we learn more about the long-term effects of LVV exposure, hopefully through federal exposure monitoring, and as 
the treatments for retrovirus exposures progress, the balance between the potential risks of insertional mutagenesis and the side effects of treatments will shift. As the risk of exposure is better defined, it may be determined that there is no benefit to treating exposures. And conversely, as the antiviral treatments become better tolerated with fewer side effects, the decision to treat may be strengthened. Either way, the topic should be revisited in the future to determine the best course of action for such exposures.

\section{ACKNOWLEDGMENTS}

Special thanks are given to Mary Ann Sondrini from the Eagleson Institute for coordinating the Lentiviral Vector Symposium. We would also like to thank Nicole Wolf for graphic design of figures.

\section{REFERENCES}

1. Rossetti M, Cavarelli M, Gregori S, Scarlatti G. HIV-derived vectors for gene therapy targeting dendritic cells. Adv Exp Med Biol. 2013;762:239-261.

2. Kafri T. Gene delivery by lentivirus vectors an overview. Methods Mol Biol. 2004;246:367-390.

3. Rothe M, Modlich U, Schambach A. Biosafety challenges for use of lentiviral vectors in gene therapy. Curr Gene Ther. 2013;13:453-468.

4. Jin C, Fotaki G, Ramachandran M, Nilsson B, Essand M, Yu D. Safe engineering of CAR $\mathrm{T}$ cells for adoptive cell therapy of cancer using long-term episomal gene transfer. EMBO Mol Med. 2016;8:702-711.

5. Gianotti-Sommer A, Rozelle SS, Sullivan S, et al. Generation of Human Induced Pluripotent Stem Cells From Peripheral Blood Using the STEMCCA Lentiviral Vector. Cambridge, MA: StemBook; 2008.

6. Ramezani A, Hawley RG. Overview of the HIV-1 lentiviral vector system. Curr Protoc Mol Biol; 2002. Chapter 16:Unit 16.21.

7. Naldini L, Blomer U, Gallay P, et al. In vivo gene delivery and stable transduction of nondividing cells by a lentiviral vector. Science. 1996; 272:263-267.

8. Zufferey R, Nagy D, Mandel RJ, Naldini L, Trono D. Multiply attenuated lentiviral vector achieves efficient gene delivery in vivo. Nat Biotechnol. 1997; $15: 871-875$

9. Kim VN, Mitrophanous K, Kingsman SM, Kingsman AJ. Minimal requirement for a lentivirus vector based on human immunodeficiency virus type 1 . J Virol. 1998:72:811-816.

10. Cockrell AS, Ma H, Fu K, McCown TJ, Kafri T. A trans-lentiviral packaging cell line for high-titer conditional self-inactivating HIV-1 vectors. Mol Ther. 2006; $14: 276-284$

11. Wu X, Wakefield JK, Liu H, et al. Development of a novel trans-lentiviral vector that affords predictable safety. Mol Ther. 2000;2:47-55.

12. Zufferey R, Dull T, Mandel RJ, et al. Self-inactivating lentivirus vector for safe and efficient in vivo gene delivery. $J$ Virol. 1998;72:9873-9880.

13. Alteri C, Soria A, Bertoli A, et al. HIV-1 Laboratory Contagion During Recombination Procedures With Defective Constructs. Boston, MA: Conference on Retroviruses and Opportunistic Infections; February 22-25, 2016.

14. De Ravin SS, Wu X, Moir S, et al. Lentiviral hematopoietic stem cell gene therapy for X-linked severe combined immunodeficiency. Sci Transl Med. 2016;8:335ra357.
15. Cavazzana-Calvo M, Hacein-Bey S, de Saint Basile G, et al. Gene therapy of human severe combined immunodeficiency (SCID)-X1 disease. Science. 2000;288:669-672.

16. Hacein-Bey-Abina S, Von Kalle C, Schmidt M, et al. LMO2-associated clonal $\mathrm{T}$ cell proliferation in two patients after gene therapy for SCID-X1. Science. 2003;302:415-419.

17. Howe SJ, Mansour MR, Schwarzwaelder K, et al. Insertional mutagenesis combined with acquired somatic mutations causes leukemogenesis following gene therapy of SCID-X1 patients. J Clin Invest. 2008;118: $3143-3150$.

18. Stein S, Ott MG, Schultze-Strasser S, et al. Genomic instability and myelodysplasia with monosomy 7 consequent to EVI1 activation after gene therapy for chronic granulomatous disease. Nat Med. 2010;16: 198-204.

19. Cavazzana-Calvo M, Payen E, Negre O, et al. Transfusion independence and HMGA2 activation after gene therapy of human beta-thalassaemia. Nature. 2010;467:318-322.

20. Occupational Safety and Health Standards: Bloodborne Pathogens: Occupational Safety \& Health Administration. [cited 05/18/2016]. Available at: https://www.osha.gov/pls/oshaweb/owadisp.show_document?p_table=S TANDARDS\&p_id=10051. Accessed September 7, 2016

21. Siegel JD, Rhinehart E, Jackson M, Chiarello L, Health Care Infection Control Practices Advisory C. 2007 Guideline for Isolation Precautions: preventing transmission of infectious agents in health care settings. Am J Infect Control. 2007;35:S65-S164.

22. Jones RM, Brosseau LM. Aerosol transmission of infectious disease. J Occup Environ Med. 2015;57:501-508.

23. Pedrosa PB, Cardoso TA. Viral infections in workers in hospital and research laboratory settings: a comparative review of infection modes and respective biosafety aspects. Int J Infect Dis. 2011;15:e366-e376.

24. Eberle J, Habermann J, Gurtler LG. HIV-1 infection transmitted by serum droplets into the eye: a case report. AIDS. 2000;14:206-207.

25. Kuhar DT, Henderson DK, Struble KA, et al. Updated US Public Health Service guidelines for the management of occupational exposures to human immunodeficiency virus and recommendations for postexposure prophylaxis. Infect Control Hosp Epidemiol. 2013;34:875-892.

26. Fonner VA, Dalglish SL, Kennedy CE, et al. Effectiveness and safety of oral HIV pre-exposure prophylaxis (PrEP) for all populations: a systematic review and meta-analysis. AIDS. 2016;30:1973-1983.

27. Final Action Under the NIH Guidelines for Research Involving Recombinant or Synthetic Nucleic Acid Molecules (NIH Guidelines). 81 Federal Register 15315. 2016;15315-15322.

28. Cronin J, Zhang XY, Reiser J. Altering the tropism of lentiviral vectors through pseudotyping. Curr Gene Ther. 2005;5:387-398.

29. Occupational Safety \& Health Administration: General Duty Clause. Available at: https://www.osha.gov/pls/oshaweb/owadisp.show_document? p_id=3359\&p_table=OSHACT. Accessed September 7, 2016.

30. 9/11 Health [05/18/2016]. Available at: http://www.nyc.gov/html/doh/wtc/ html/home/home.shtml. Accessed May 28, 2016.

31. Building a Safety Program to Protect the Nanotechnology Workforce: A Guide for Small to Medium-Sized Enterprises. Washington DC: National Institute for Occupational Safety and Health; 2016.

32. Schulte PA, Mundt DJ, Nasterlack M, Mulloy KB, Mundt KA. Exposure registries: overview and utility for nanomaterial workers. J Occup Environ Med. 2011;53:S42-S47. 


\section{Appendix 1: An Example of a Laboratory-Specific ERP for a Lentiviral Vector System to Induce c-Raf Expression}

\section{Laboratory-Specific Lentivirus Exposure Response Plan}

\section{Biological Agent Information}

Lentiviral vectors are single-stranded RNA viral vectors derived from the human immunodeficiency virus (HIV) that retain the ability to integrate into the genome of infected cells.

\section{Vector System}

- pRSV-Rev (packaging Vector)

- pLKO.3G (transfer Vector, eGFP marker)

- pMD2.G (VSV-g envelope vector)

- 293T Human Cell line

\section{Transgene Information}

- $c-\operatorname{Raf}$ (an oncogene that is part of the cellular growth pathway)

- Mutants of $c$-Raf with unknown function

\section{Risks of Exposure}

- Insertional mutagenesis-the lentiviral vector can disrupt the normal regulation of cell development and proliferation leading to oncogenesis.

- Transgene oncogenesis - the transgene is an oncogene and may induce oncogenesis in infected cells.

- Generation of replication-competent retrovirus-the lentiviral vector may undergo a series of low probability events to revert to a replication-competent retrovirus.

\section{Routes of Transmission}

- Direct parenteral inoculation

- Contact with mucous membranes or nonintact skin

- Direct contact at a close range to droplets from an aerosolgenerating procedure outside of primary containment

\section{Response}

- Intact Skin Exposure: immediately wash off the affected area with copious amounts of running water to dilute, cleanse, and flush the LVVs from intact skin.

- Nonintact Skin Exposure: immediately wash the exposed area with copious amounts of soap and water to dilute, cleanse, and flush the LVVs from the area.

- Mucous Membrane Exposure [Eye(s), Nose, or Mouth]: immediately flush the area with running water for at least 15 minutes.

- Droplet Exposure: see above based upon the area exposed

\section{First Aid Treatment}

- Call emergency personnel if immediate medical care is needed (911)

- Stabilize the individual and provide first aid for injuries that require immediate medical care (eg, deep cuts, bleeding, etc.)

Postexposure Prophylaxis

- Based upon discussion before the incident, the following treatments may be offered.

- As soon as possible but within 72 hours, initiate a 7-day course of a NRTI (such as tenofovir) and an integrase inhibitor (such as raltegravir or dolutegravir).

- Observation and treatment of overt effects of the exposure incident.

- If no prior discussion about treatment has occurred, treatment with tenofovir and raltegravir/dolutegravir should be considered and begin within 72 hours and continued based upon a discussion and acceptance of the risks and benefits of treatment.

\section{Reporting Incident}

- All exposure incidents should immediately be reported to the principal investigator, the Biosafety office, or Occupational Health.

- All exposures to biological agents should be reported to the Institutional Biosafety Committee.

- If the exposure results in an overt exposure to recombinant or synthetic nucleic acid molecules will be reported to the NIH Office of Biotechnology Activities

- All sharps accidents will be reported on the OSHA Log of WorkRelated. 